\title{
LARGE TIME BEHAVIOR IN A DENSITY-DEPENDENT POPULATION DYNAMICS PROBLEM WITH AGE STRUCTURE AND CHILD CARE
}

\author{
VLADAS SKAKAUSKAS \\ Faculty of Mathematics and Informatics, Vilnius University \\ Naugarduko 24, 03225, Vilnius, Lithuania \\ E-mail: vladas.skakauskas@maf.vu.lt
}

\begin{abstract}
Two asexual density-dependent population dynamics models with age-dependence and child care are presented. One of them includes the random diffusion while in the other the population is assumed to be non-dispersing. The population consists of the young (under maternal care), juvenile, and adult classes. Death moduli of the juvenile and adult classes in both models are decomposed into the sum of two terms. The first presents death rate by the natural causes while the other describes the environmental influence depending on the total density of the juvenile and adult individuals. An existence and uniqueness theorem is proved, a class of separable solutions is constructed, and the large time behavior of the general and separable solutions is given for the non-dispersing population with stationary vital rates. The steady-state and separable solutions are constructed and the large time behavior of the separable solutions is studied for the population with the spatial dispersal.
\end{abstract}

1. Introduction. The Sharpe-Lotka-McKendrick-von Foerster (see, e.g., [12]) and Hoppensteadt-Staroverov-Hadeler (see, e.g., [4]) models are well known in mathematical biology. The first one (or its Gurtin-MacCamy modification [3]) is usually used to describe the dynamics of asexual age-structured populations. The other one is the basic model in mathematical demography and describes the evolution of populations forming permanent pairs. Both models do not treat the child care phenomenon. Moss de Oliveira (see [8] and references therein) was the first to study consequences of this phenomenon. She introduced parental care into the Penna bit-string model (see, e.g., [8]) which is well known in the computer simulations of biological ageing. In asexual version of this model each individual is represented by a computer string of 32 bits. In sexual version each individual has two bit strings. Some results of biological ageing obtained by using

2000 Mathematics Subject Classification: Primary 92D25; Secondary 92D40.

Key words and phrases: population dynamics, age-structured population, child care.

The paper is in final form and no version of it will be published elsewhere. 
Monte Carlo simulations for the Penna model are reviewed in [8]. In particular, ageing of a semelparous species (i.e., a species that reproduce only once in life) and the spatial distribution of the populations are studied.

In [11] we proposed two non-dispersing population dynamics models with age structure and maternal care: one for bisexual populations without formation of permanent pairs and the other for asexual ones producing offspring at fixed ages. In contrast to the Penna aging model those models are represented by systems of PDE (or integro-PDE) equations and can be examined analytically.

In the present paper we generalize the model in [11] for the asexual population and present two asexual population dynamics models taking into account age structure, child care, and a population density factor describing an environmental influence. Both models are represented by systems of integro-PDE. One of the models includes random diffusion in a bounded domain with an extremely inhospitable boundary (or without migration across it) while in the other the population is assumed to be non-dispersing. In both cases the population consists of the young, juvenile, and adult classes. The young and juvenile individuals are of pre-reproductive age, while the adults have reproductive and post-reproductive age grades. Ages of production of offspring are not fixed in theses models. We assume that all young individuals are under maternal care and all adults of reproductive age are divided into fertile singles (having no offspring under maternal care at the given time), mothers (taking care of offspring), and temporarily sterile singles (individuals for whom child care time is over but whose age still is in the sterility interval after delivery). We also assume that young offspring and their mothers are at the same position at every time and, in the case of the dispersing population, move together with them. We let the vital rates of the juvenile and adult individuals depend on their total density $N$ and, following Moss de Oliveira [8], we assume that young offspring are subject to natural mortality and are protected from density related increases of mortality dependent on $N$ directly. We also adopt the strong child care law by which all young offspring are killed if their mother dies. Therefore, the death rate of young individuals consists of their natural death rate independent of $N$ plus the death rate of their mothers. Such the division of population is natural for some species of mammals, e.g. the European polecat species (mustela putorius) living in England. In particular, these models can be applied to describe the evolution of semelparous species.

The description of the long time behavior of the population subject to the models below is one of goals of this paper.

The paper is organized as follows. In Sec. 3, which consists of three subsections, we present and analyze a non-dispersing population dynamics model. In 3.1 separable solutions and their large time behavior are studied. In 3.2 we prove an existence and uniqueness theorem, and in 3.3 we give the large time behavior of the general solution in the case of stationary vital rates. Section 4 is devoted to the dynamics of a dispersing population and consists of two subsections. In 4.1 the separable solutions and their large time asymptotics are examined, and in 4.2 the steady-state solutions are studied. A discussion follows in Sec. 5, including some comments about the models. 
2. Notation. The following notation is used for the analysis of the population dynamics with child care.

$\mathbb{R}^{m}$ : the Euclidean space of dimension $m$ with $x=\left(x_{1}, \ldots, x_{m}\right)$,

$\kappa:$ the diffusion modulus,

$T_{0}, h, T_{1},\left(T_{0}<h<T_{1}\right)$ : the child care period, sterility period after delivery, and maturation period, respectively,

$\left(0, T_{1}\right)=\left(0, T_{0}\right) \cup\left[T_{0}, T_{1}\right)$ : the pre-reproductive age interval which consists of $\left(0, T_{0}\right)$ (the young) and $\left[T_{0}, T_{1}\right.$ ) (the juvenile) age intervals,

$\left[T_{1}, \infty\right)$ : the adult age interval,

$T_{i+1}=T_{1}+i h, i=0,1, \ldots, n$ : age of production of the feasible $(i+1)$ st generation,

$\sigma_{1}=\left(T_{1}, T_{n+1}\right), \bar{\sigma}_{1}=\left[T_{1}, T_{n+1}\right]:$ the delivery age interval,

$\left(T_{1}, T_{n+1}+T_{0}\right)$ : the child care age interval,

$\left(T_{n+2}, \infty\right), T_{n+2}=T_{n+1}+h$ : the post-reproductive age interval,

$\sigma_{2}=\left(T_{2}, T_{n+2}\right), \bar{\sigma}_{2}=\left[T_{2}, T_{n+2}\right]$,

$\sigma=\left(T_{1}+T_{0}, T_{n+1}+T_{0}\right), \bar{\sigma}=\left[T_{1}+T_{0}, T_{n+1}+T_{0}\right]$,

$D_{1}=\left(T_{0}, \infty\right), \bar{D}_{1}=\left[T_{0}, \infty\right), \tilde{D}_{1}=\bar{D}_{1} \backslash\left\{T_{1}, T_{2}, T_{n+1}, T_{n+2}\right\}$,

$D_{2}=\left\{\left(\tau_{1}, \tau_{2}\right): \tau_{2} \in\left(0, T_{0}\right), \tau_{1} \in\left(T_{1}+\tau_{2}, T_{n+1}+\tau_{2}\right)\right\}$,

$\bar{D}_{2}=\left\{\left(\tau_{1}, \tau_{2}\right): \tau_{2} \in\left[0, T_{0}\right], \tau_{1} \in\left[T_{1}+\tau_{2}, T_{n+1}+\tau_{2}\right]\right\}$,

$\tilde{D}_{2}=\bar{D}_{2} \backslash\left\{\left(\tau_{1}, \tau_{2}\right): \tau_{1}-\tau_{2}=T_{2}\right\}$,

$D_{3}=\left\{\left(\tau_{1}, \tau_{3}\right): \tau_{3} \in\left(0, h-T_{0}\right), \tau_{1} \in\left(T_{1}+T_{0}+\tau_{3}, T_{n+1}+T_{0}+\tau_{3}\right)\right\}$,

$\bar{D}_{3}=\left\{\left(\tau_{1}, \tau_{3}\right): \tau_{3} \in\left[0, h-T_{0}\right], \tau_{1} \in\left[T_{1}+T_{0}+\tau_{3}, T_{n+1}+T_{0}+\tau_{3}\right]\right\}$,

$\tilde{D}_{3}=\bar{D}_{3} \backslash\left\{\left(\tau_{1}, \tau_{3}\right): \tau_{1}-\tau_{3}=T_{2}+T_{0}\right\}$,

$\partial_{t}=\partial / \partial t, \partial_{\tau_{i}}=\partial / \partial \tau_{i}$,

$\mathfrak{D}_{1}=\partial_{t}+\partial_{\tau_{1}}, \mathfrak{D}_{2}=\partial_{t}+\partial_{\tau_{1}}+\partial_{\tau_{2}}, \mathfrak{D}_{3}=\partial_{t}+\partial_{\tau_{1}}+\partial_{\tau_{3}}$,

$C^{0}(\bar{D})$ (resp. $\left.C^{0}(D)\right)$ : the class of bounded continuous functions in $\bar{D}$ (resp. $D$ ), where $D$ is a domain not necessarily bounded and $\bar{D}$ the closure of $D$,

$C^{1}(\bar{D})$ (resp. $C^{1}(D)$ ): a class of bounded continuous functions $f\left(x_{1}, \ldots, x_{m}\right)$ in $\bar{D}$ (resp. $D)$ with $\partial_{x_{i}} f \in C^{0}(\bar{D})\left(\operatorname{resp} . C^{0}(D)\right), i=1, \ldots, m$,

$C^{0,1, \ldots, 0}(D)$ : the class of bounded continuous functions $f\left(x_{1}, \ldots, x_{m}\right)$ in $D$ with $\partial_{x_{2}} f \in$ $C^{0}(D)$,

$L^{p}(D),(p=1,2)$ : the standard Banach space of functions $f$ with norm $\|f\|=$ $\left(\int_{D}|f(x)|^{p} d x\right)^{1 / p}$.

$u_{1}\left(t, \tau_{1}, x\right)$ : the age-space-density of individuals aged $\tau_{1}$ at time $t$ at the position $x$ who are of juvenile, fertile single, or post-reproductive age,

$u_{2}\left(t, \tau_{1}, \tau_{2}, x\right)$ : the age-space-density of young offspring aged $\tau_{2}$ at time $t$ at the position $x$ whose mothers are of age $\tau_{1}$ at the same time $t$,

$u_{3}\left(t, \tau_{1}, \tau_{2}, x\right)$ : the age-space-density of individuals aged $\tau_{1}$ at time $t$ at the position $x$ who care for offspring aged $\tau_{2}$ at the same time $t$,

$u_{4}\left(t, \tau_{1}, \tau_{3}, x\right)$ : the age-space-density of individuals aged $\tau_{1}$ at time $t$ at the position $x$ for whom child care time is over but who still have been in the temporarily sterile class for $\tau_{3}$ units of time (i.e. for whom time $\tau_{3}$ is passed after the end of child care),

$N(t, x)$ : the total spatial density of the juvenile and adult individuals at time $t$ at the location $x$, 
$\alpha\left(t, \tau_{1}, x, N\right) d t$ : the probability for individuals aged $\tau_{1}$ at time $t$ to produce offspring at the location $x$ in the time interval $[t, t+d t]$,

$\alpha\left(t, \tau_{1}, x, N\right) b\left(t, \tau_{1}, x, N\right)$ : the birth rate of offspring at time $t$ at the location $x$ whose mothers are of age $\tau_{1}$ at the same time $t$,

$\nu_{1}\left(t, \tau_{1}, x, N\right)$ : the death rate of individuals aged $\tau_{1}$ at time $t$ at the position $x$ who are of juvenile, fertile single, or post-reproductive age,

$\nu_{2}\left(t, \tau_{1}, \tau_{2}, x\right)$ : the death rate of young offspring aged $\tau_{2}$ at time $t$ at the location $x$ whose mothers are of age $\tau_{1}$ at the same time $t$,

$\nu_{3}\left(t, \tau_{1}, \tau_{2}, x, N\right)$ : the death rate of individuals aged $\tau_{1}$ at time $t$ at the location $x$ who care for offspring aged $\tau_{2}$ at the same time $t$,

$\nu_{4}\left(t, \tau_{1}, \tau_{3}, x, N\right)$ : the death rate of individuals aged $\tau_{1}$ at time $t$ at the position $x$ for whom child care time is over but who still have been in the temporarily sterile class for $\tau_{3}$ units of time,

$\left[\left.u_{1}\right|_{\tau_{1}=T_{s}}\right]$ : a jump discontinuity of $u_{1}$ at $\tau_{1}=T_{s}$.

In what follows the child care period $T_{0}$, sterility period after delivery $h$, and maturation period $T_{1}$ are assumed to be constant. In the case of non-dispersing populations all functions $u_{1}, \ldots, u_{4}, \nu_{1}, \ldots, \nu_{4}, b, \alpha, u_{10}, \ldots, u_{30}$, and $u_{40}$ do not depend on the spatial position $x$.

3. The non-dispersing population dynamics model. Using the balance law, we derive the following density-dependent population dynamics model with child care:

$$
\begin{aligned}
& \left\{\begin{array}{l}
\mathfrak{D}_{1} u_{1}=-\nu_{1}\left(t, \tau_{1}, N\right) u_{1}- \\
\left\{\begin{array}{l}
0, \tau_{1} \notin \bar{\sigma}_{1}, \\
\alpha\left(t, \tau_{1}, N\right) u_{1}, \tau_{1} \in \sigma_{1}
\end{array}+\left\{\begin{array}{l}
0, \tau_{1} \notin \bar{\sigma}_{2}, \\
\left.u_{4}\right|_{\tau_{3}=h-T_{0}}, \tau_{1} \in \sigma_{2},
\end{array} \tau_{1} \in D_{1}, t>0\right.\right. \\
\left.u_{1}\right|_{\tau_{1}=T_{0}}=\left.\int_{\sigma} u_{2}\right|_{\tau_{2}=T_{0}} d \tau_{1}, t \geq 0 \\
\left.u_{1}\right|_{t=0}=u_{10}, \tau_{1} \in \bar{D}_{1} \\
{\left[\left.u_{1}\right|_{\tau_{1}=T_{s}}=0, t \geq 0, s=1,2, n+1, n+2 ;\right.}
\end{array}\right. \\
& \left\{\begin{array}{l}
\mathfrak{D}_{2} u_{2}=-\left(\nu_{2}\left(t, \tau_{1}, \tau_{2}\right)+\nu_{3}\left(t, \tau_{1}, \tau_{2}, N\right)\right) u_{2},\left(\tau_{1}, \tau_{2}\right) \in D_{2}, t>0 \\
\left.u_{2}\right|_{\tau_{2}=0}=\alpha\left(t, \tau_{1}, N\right) b\left(t, \tau_{1}, N\right) u_{1}\left(t, \tau_{1}\right), \tau_{1} \in \bar{\sigma}_{1}, t \geq 0 \\
\left.u_{2}\right|_{t=0}=u_{20} \text { in } \bar{D}_{2}
\end{array}\right.
\end{aligned}
$$

$$
\begin{gathered}
\left\{\begin{array}{l}
\mathfrak{D}_{2} u_{3}=-\nu_{3}\left(t, \tau_{1}, \tau_{2}, N\right) u_{3},\left(\tau_{1}, \tau_{2}\right) \in D_{2}, t>0, \\
\left.u_{3}\right|_{\tau_{2}=0}=\alpha\left(t, \tau_{1}, N\right) u_{1}\left(t, \tau_{1}\right), \tau_{1} \in \bar{\sigma}_{1}, t \geq 0, \\
\left.u_{3}\right|_{t=0}=u_{30} \text { in } \bar{D}_{2} ;
\end{array}\right. \\
\left\{\begin{array}{l}
\mathfrak{D}_{3} u_{4}=-\nu_{4}\left(t, \tau_{1}, \tau_{3}, N\right) u_{4},\left(\tau_{1}, \tau_{3}\right) \in D_{3}, t>0, \\
\left.u_{4}\right|_{\tau_{3}=0}=\left.u_{3}\right|_{\tau_{2}=T_{0}}, \tau_{1} \in \bar{\sigma}, t \geq 0, \\
\left.u_{4}\right|_{t=0}=u_{40} \text { in } \bar{D}_{3} ;
\end{array}\right. \\
N=\int_{D_{1}} u_{1} d \tau_{1}+\int_{D_{2}} u_{3} d \tau_{1} d \tau_{2}+\int_{D_{3}} u_{4} d \tau_{1} d \tau_{3}, t \geq 0 .
\end{gathered}
$$


We stress that $\nu_{2}$ is $N$-independent and add the following compatibility conditions:

$$
\begin{aligned}
\left\{\begin{array}{l}
u_{10}\left(T_{0}\right)=\int_{\sigma} u_{20}\left(\tau_{1}, T_{0}\right) d \tau_{1}, \\
u_{20}\left(\tau_{1}, 0\right)=\alpha\left(0, \tau_{1}, N_{0}\right) u_{10}\left(\tau_{1}\right) b\left(0, \tau_{1}, N_{0}\right) \text { in } \bar{\sigma}_{1}, \\
u_{30}\left(\tau_{1}, 0\right)=\alpha\left(0, \tau_{1}, N_{0}\right) u_{10}\left(\tau_{1}\right) \text { in } \bar{\sigma}_{1}, \\
u_{40}\left(\tau_{1}, 0\right)=u_{30}\left(\tau_{1}, T_{0}\right) \text { in } \bar{\sigma},
\end{array}\right. \\
N_{0}=\int_{D_{1}} u_{10} d \tau_{1}+\int_{D_{2}} u_{30} d \tau_{1} d \tau_{2}+\int_{D_{3}} u_{40} d \tau_{1} d \tau_{3}, t \geq 0 .
\end{aligned}
$$

As follows from the foregoing, the given constants $T_{0}, h, T_{1}$ and functions $b, \alpha, \nu_{1}$, $\ldots, \nu_{4}, u_{10}\left(\tau_{1}\right), u_{20}\left(\tau_{1}, \tau_{2}\right), u_{30}\left(\tau_{1}, \tau_{2}\right)$, and $u_{40}\left(\tau_{1}, \tau_{3}\right)$, as well as the unknown ones $u_{1}, u_{2}$, $u_{3}$, and $u_{4}$ must be positive, otherwise they have no biological significance. In this paper we shall consider the case where $T_{0}<h<T_{1}$. This assumption is natural for some species of populations. Starting with the initial distributions $u_{10}, \ldots, u_{40}$ we want to describe the evolution of $u_{1}, u_{2}, u_{3}$, and $u_{4}$ satisfying (3.1)-(3.7).

3.1. Separable solutions. In this section we shall examine model (3.1)-(3.7) with

$$
\left\{\begin{array}{l}
\alpha=\alpha\left(\tau_{1}\right), b=b\left(\tau_{1}\right), \nu_{1}=\mu_{1}\left(\tau_{1}\right)+\rho(N), \nu_{2}=\mu_{2}\left(\tau_{1}, \tau_{2}\right), \\
\nu_{3}=\mu_{3}\left(\tau_{1}, \tau_{2}\right)+\rho(N), \nu_{4}=\mu_{4}\left(\tau_{1}, \tau_{3}\right)+\rho(N)
\end{array}\right.
$$

We look for solutions in the form

$$
u_{i}=f(t) u_{i 0}, u_{i 0}=U v_{i} \exp \left\{\lambda\left(T_{0}-\tau_{1}\right)\right\}, i=1,2,3,4, v_{1}\left(T_{0}\right)=1, f(0)=1,
$$

where $U$ is a positive constant and $f(t), v_{1}\left(\tau_{1}\right), v_{2}\left(\tau_{1}, \tau_{2}\right), v_{3}\left(\tau_{1}, \tau_{2}\right), v_{4}\left(\tau_{1}, \tau_{3}\right)$ and the constant $\lambda$ are to be determined.

THEOREM 1. Let constants $T_{0}<h<T_{1}$ and functions $\mu_{1}, \ldots, \mu_{4}, \alpha$, and $b$ be positive and: $\rho \in C^{1}([0, \infty)), \rho>0$ and $\rho^{\prime}>0$ for all $N>0, \alpha, b \in C^{1}\left(\bar{\sigma}_{1}\right), \mu_{1} \in C^{0}\left(\bar{D}_{1}\right)$, $\mu_{2}, \mu_{3} \in C^{1,0}\left(\bar{D}_{2}\right), \mu_{4} \in C^{1,0}\left(\bar{D}_{3}\right)$. Assume that $\lambda_{0}$ is the unique real root of the equation

$$
R(\lambda)=1, R(\lambda):=\int_{\sigma} v_{2}\left(\tau_{1}, T_{0}\right) \exp \left\{\lambda\left(T_{0}-\tau_{1}\right)\right\} d \tau_{1}
$$

satisfying the condition

$$
\int_{D_{1}} \exp \left\{-\lambda_{0} x-\int_{T_{0}}^{x} \mu_{1}(\xi) d \xi\right\} d x<\infty .
$$

Then problem (3.1)-(3.7) and (3.1.1) has a one-parameter class of nonnegative separable solutions of type (3.1.2) such that:

$$
\begin{aligned}
& u_{1} \in C^{0}\left([0, \infty) \times \bar{D}_{1}\right) \cap C^{1}\left([0, \infty) \times \tilde{D}_{1}\right), \\
& u_{s} \in C^{0}\left([0, \infty) \times \bar{D}_{2}\right) \cap C^{1}\left([0, \infty) \times \tilde{D}_{2}\right), s=2,3, \\
& u_{4} \in C^{0}\left([0, \infty) \times \bar{D}_{3}\right) \cap C^{1}\left([0, \infty) \times \tilde{D}_{3}\right),
\end{aligned}
$$

and, for $i=1,2,3,4$,

$$
\begin{aligned}
& u_{i} \underset{t \rightarrow \infty}{\longrightarrow} 0 \text { if } \lambda_{0} \leq \rho(0), \\
& u_{i} \underset{t \rightarrow \infty}{\longrightarrow} \infty \text { if } \sup _{N \geq 0} \rho(N) \leq \lambda_{0}<\infty, \\
& u_{i} \underset{t \rightarrow \infty}{\longrightarrow} u_{i 0} N_{*} / N_{0} \text { with } N_{0} \text { defined by (3.7) if } \lambda_{0}>0 \text { and there exists } N_{*}>0 \text { such }
\end{aligned}
$$

that $\rho\left(N_{*}\right)=\lambda_{0}$. 
Proof. By direct substitution of (3.1.2) into (3.1)-(3.5) we get (3.1.3) and equations:

$$
\begin{gathered}
N=f N_{0} \\
N^{\prime}=N\left(\lambda_{0}-\rho(N)\right), N(0)=N_{0} \\
\left\{\begin{array}{c}
\partial_{\tau_{1}} v_{2}+\partial_{\tau_{2}} v_{2}=-v_{2} \mu_{23}\left(\tau_{1}, \tau_{2}\right) \text { in } D_{2}, v_{2}\left(\tau_{1}, 0\right)=\alpha\left(\tau_{1}\right) b\left(\tau_{1}\right) v_{1}\left(\tau_{1}\right), \tau_{1} \in \bar{\sigma}_{1}, \\
\partial_{\tau_{1}} v_{3}+\partial_{\tau_{2}} v_{3}=-v_{3} \mu_{3} \text { in } D_{2}, v_{3}\left(\tau_{1}, 0\right)=\alpha\left(\tau_{1}\right) v_{1}\left(\tau_{1}\right), \tau_{1} \in \bar{\sigma}_{1} \\
\partial_{\tau_{1}} v_{4}+\partial_{\tau_{3}} v_{4}=-v_{4} \mu_{4} \text { in } D_{3}, v_{4}\left(\tau_{1}, 0\right)=v_{3}\left(\tau_{1}, T_{0}\right), \tau_{1} \in \bar{\sigma}
\end{array}\right.
\end{gathered}
$$

$$
v_{1}^{\prime}=-\mu_{1} v_{1}-\left\{\begin{array}{l}
0, \tau_{1} \notin \bar{\sigma}_{1}, \\
\alpha\left(\tau_{1}\right) v_{1}, \tau_{1} \in \sigma_{1}
\end{array}+\left\{\begin{array}{l}
0, \tau_{1} \notin \bar{\sigma}_{2}, \\
v_{4}\left(\tau_{1}, h-T_{0}\right), \tau_{1} \in \sigma_{2}
\end{array} \quad \text { in } D_{1}, v_{1}\left(T_{0}\right)=1\right.\right.
$$

$\left[v_{1}\left(T_{s}\right)\right]=0, s=1,2, n+1, n+2$. Here and in what follows the prime superscript indicates differentiation and $\mu_{23}=\mu_{2}+\mu_{3}$. Note that $N_{0}$ depends on $\lambda_{0}$. Integration of (3.1.7) yields

$$
\begin{gathered}
v_{2}=\left.\left(b \alpha v_{1}\right)\right|_{\left(\tau_{1}-\tau_{2}\right)} \exp \left\{-\int_{0}^{\tau_{2}} \mu_{23}\left(x+\tau_{1}-\tau_{2}, x\right) d x\right\}, \\
v_{3}=\left.\left(\alpha v_{1}\right)\right|_{\left(\tau_{1}-\tau_{2}\right)} \exp \left\{-\int_{0}^{\tau_{2}} \mu_{3}\left(x+\tau_{1}-\tau_{2}, x\right) d x\right\}, \\
v_{4}=\left.\left(\alpha v_{1}\right)\right|_{\left(\tau_{1}-\tau_{3}-T_{0}\right)} \exp \left\{-\int_{0}^{T_{0}} \mu_{3}\left(x+\tau_{1}-\tau_{3}-T_{0}, x\right) d x-\right. \\
\left.\int_{0}^{\tau_{3}} \mu_{4}\left(x+\tau_{1}-\tau_{3}, x\right) d x\right\} .
\end{gathered}
$$

Observe that Eqs. (3.1.8) and (3.1.11) can be easily solved. Substituting (3.1.9) into (3.1.3), we get

$$
1=\int_{\sigma_{1}} \alpha(\xi) b(\xi) v_{1}(\xi) \exp \left\{-\xi \lambda-\int_{0}^{T_{0}} \mu_{23}(x+\xi, x) d x\right\} d \xi
$$

The distribution of roots of this characteristic equation is well known. Let $\lambda_{i}, i=1,2, \ldots$ be the complex roots. Then $\operatorname{Re} \lambda_{i}<\lambda_{0}$, where $\lambda_{0}$ is a unique real root. Since $0 \leq \rho \in$ $C^{1}([0, \infty))$, Eq. (3.1.6) has a unique global solution $N \leq N_{0} \exp \left\{\lambda_{0} t\right\}$. Its long time behavior is given by Langlais and Milner in [6]. This completes the proof.

3.2. The existence and uniqueness theorem. In this section we examine problem (3.1)(3.7) in the case where

$$
\left\{\begin{array}{l}
\alpha=\alpha\left(t, \tau_{1}\right), b=b\left(t, \tau_{1}, N\right), \nu_{1}=\mu_{1}\left(t, \tau_{1}\right)+\rho(N), \nu_{2}=\mu_{2}\left(t, \tau_{1}, \tau_{2}\right), \\
\nu_{3}=\mu_{3}\left(t, \tau_{1}, \tau_{2}\right)+\rho(N), \nu_{4}=\mu_{4}\left(t, \tau_{1}, \tau_{3}\right)+\rho(N)
\end{array}\right.
$$

Set

$$
\begin{aligned}
& Q_{1}=\left\{\left(t, \tau_{1}\right): t=\tau_{1}, \tau_{1}-T_{0}, \tau_{1}-T_{1}, \tau_{1}-T_{2}, \tau_{1}-T_{n+1}, \tau_{1}-T_{n+2}, \tau_{1}=T_{1}, T_{2},\right. \\
& \left.T_{n+1}, T_{n+2}\right\} \\
& Q_{2}=\left\{\left(t, \tau_{1}, \tau_{2}\right): \tau_{1}-\tau_{2}=T_{2}, t=\tau_{2}, \tau_{1}, \tau_{1}-T_{0}, \tau_{1}-T_{1}, \tau_{1}-T_{2}, \tau_{1}-T_{n+1}\right\} \\
& Q_{3}=\left\{\left(t, \tau_{1}, \tau_{3}\right): \tau_{1}-\tau_{3}=T_{2}+T_{0}, t=\tau_{3}, \tau_{3}+T_{0}, \tau_{1}, \tau_{1}-T_{0}, \tau_{1}-T_{1}, \tau_{1}-T_{2}, \tau_{1}-T_{n+1}\right\} .
\end{aligned}
$$

THEOREM 2. Let functions $\alpha, b, \mu_{1}, \ldots, \mu_{4}, u_{10}, \ldots, u_{40}$ and constants $T_{0}<h<T_{1}$ be positive and: $\rho \in C^{1}([0, \infty)), \rho>0$ and $\rho^{\prime}>0$ for all $N>0, \alpha \in C^{1}\left([0, \infty) \times \bar{\sigma}_{1}\right), b \in$ 
$C^{1}\left([0, \infty) \times \bar{\sigma}_{1} \times[0, \infty)\right), \mu_{1} \in C^{1,0}\left([0, \infty) \times \bar{D}_{1}\right), \mu_{2}, \mu_{3} \in C^{1,1,0}\left([0, \infty) \times \bar{D}_{2}\right), \mu_{4} \in$ $C^{1,1,0}\left([0, \infty) \times \bar{D}_{3}\right), u_{10} \in C^{1}\left(\bar{D}_{1}\right) \cap L^{1}\left(D_{1}\right)$ and $u_{10}^{\prime} \in L^{1}\left(D_{1}\right), u_{20}, u_{30} \in C^{1}\left(\bar{D}_{2}\right), u_{40} \in$ $C^{1}\left(\bar{D}_{3}\right)$. Then, for any fixed $t_{*}>0$, problem (3.1)-(3.7) and (3.2.1) has a unique positive solution such that

$$
\begin{aligned}
& u_{1} \in C^{0}\left(\left[0, t_{*}\right] \times \bar{D}_{1}\right) \cap C^{1}\left(\left(\left[0, t_{*}\right] \times \bar{D}_{1}\right) \backslash Q_{1}\right), \\
& u_{s} \in C^{0}\left(\left[0, t_{*}\right] \times \bar{D}_{2}\right) \cap C^{1}\left(\left(\left[0, t_{*}\right] \times \bar{D}_{2}\right) \backslash Q_{2}\right), s=2,3, \\
& u_{4} \in C^{0}\left(\left[0, t_{*}\right] \times \bar{D}_{3}\right) \cap C^{1}\left(\left(\left[0, t_{*}\right] \times \bar{D}_{3}\right) \backslash Q_{3}\right), N \in C^{1}\left(\left[0, t_{*}\right]\right) .
\end{aligned}
$$

Proof. We stress that jumps of all partial derivatives of $u_{1}, \ldots, u_{4}$ at the sets of their discontinuity are bounded. Assuming that

$$
\left\{\begin{array}{l}
u_{1}=f(t) U_{1}\left(t, \tau_{1}\right), u_{2}=f(t) U_{2}\left(t, \tau_{1}, \tau_{2}\right), u_{3}=f(t) U_{3}\left(t, \tau_{1}, \tau_{2}\right), \\
u_{4}=f(t) U_{4}\left(t, \tau_{1}, \tau_{3}\right), f(0)=1
\end{array}\right.
$$

and substituting for $u_{1}, u_{2}, u_{3}$, and $u_{4}$ in (3.1)-(3.5), we get:

$$
\begin{gathered}
\beta(t)=\int_{D_{1}} U_{1} d \tau_{1}+\int_{D_{2}} U_{3} d \tau_{1} d \tau_{2}+\int_{D_{3}} U_{4} d \tau_{1} d \tau_{3}, \\
N^{\prime}=N\left(\beta^{\prime} / \beta-\rho(N)\right), N(0)=N_{0}
\end{gathered}
$$

with $N_{0}$ defined by (3.7),

$$
\begin{gathered}
f=N / \beta \\
\left\{\begin{array}{l}
\mathfrak{D}_{1} U_{1}=-\mu_{1} U_{1}-\left\{\begin{array}{l}
0, \tau_{1} \notin \bar{\sigma}_{1}, \\
\alpha U_{1}, \tau_{1} \in \sigma_{1}
\end{array}+\left\{\begin{array}{l}
0, \tau_{1} \notin \bar{\sigma}_{2}, \\
\left.U_{4}\right|_{\tau_{3}=h-T_{0}}, \tau_{1} \in \sigma_{2},
\end{array} \tau_{1} \in D_{1}, t>0\right.\right. \\
\left.U_{1}\right|_{\tau_{1}=T_{0}}=\left.\int_{\sigma} U_{2}\right|_{\tau_{2}=T_{0}} d \tau_{1}, t \geq 0 \\
\left.U_{1}\right|_{t=0}=u_{10} \text { in } \bar{D}_{1}, \\
{\left[\left.U_{1}\right|_{\tau_{1}=T_{s}}\right]=0, s=1,2, n+1, n+2, t \geq 0}
\end{array}\right. \\
\left\{\begin{array}{l}
\mathfrak{D}_{2} U_{2}=-\mu_{23} U_{2},\left(\tau_{1}, \tau_{2}\right) \in D_{2}, t>0 \\
\left.U_{2}\right|_{\tau_{2}=0}=\alpha b\left(t, \tau_{1}, N\right) U_{1}, \tau_{1} \in \bar{\sigma}_{1}, t \geq 0 \\
\left.U_{2}\right|_{t=0}=u_{20} \text { in } \bar{D}_{2}
\end{array}\right.
\end{gathered}
$$$$
\left\{\begin{array}{l}
\mathfrak{D}_{2} U_{3}=-\mu_{3} U_{3},\left(\tau_{1}, \tau_{2}\right) \in D_{2}, t>0 \\
\left.U_{3}\right|_{\tau_{2}=0}=\alpha U_{1}, \tau_{1} \in \bar{\sigma}_{1}, t \geq 0 \\
\left.U_{3}\right|_{t=0}=u_{30} \text { in } \bar{D}_{2}
\end{array}\right.
$$$$
\left\{\begin{array}{l}
\mathfrak{D}_{3} U_{4}=-\mu_{4} U_{4},\left(\tau_{1}, \tau_{3}\right) \in D_{3}, t>0 \\
\left.U_{4}\right|_{\tau_{3}=0}=\left.U_{3}\right|_{\tau_{2}=T_{0}}, \tau_{1} \in \bar{\sigma}, t \geq 0 \\
\left.U_{4}\right|_{t=0}=u_{40} \text { in } \bar{D}_{3}
\end{array}\right.
$$

Integration of Eqs. (3.2.6)-(3.2.9) along characteristics yields:

$(3.2 .10) U_{2}=\left\{\begin{array}{l}u_{20}\left(\tau_{1}-t, \tau_{2}-t\right) \exp \left\{-\int_{\tau_{2}-t}^{\tau_{2}} \mu_{23}\left(x+t-\tau_{2}, x+\tau_{1}-\tau_{2}, x\right) d x\right\}, t \leq \tau_{2}, \\ \left.\left(\alpha U_{1}\right)\right|_{\left(t-\tau_{2}, \tau_{1}-\tau_{2}\right)} b\left(t-\tau_{2}, \tau_{1}-\tau_{2}, N\left(t-\tau_{2}\right)\right) \times \\ \exp \left\{-\int_{0}^{\tau_{2}} \mu_{23}\left(x+t-\tau_{2}, x+\tau_{1}-\tau_{2}, x\right) d x\right\}, t \geq \tau_{2} ;\end{array}\right.$ 
$(3.2 .11)$

$$
\begin{gathered}
U_{3}=\left\{\begin{array}{l}
u_{30}\left(\tau_{1}-t, \tau_{2}-t\right) \exp \left\{-\int_{\tau_{2}-t}^{\tau_{2}} \mu_{3}\left(x+t-\tau_{2}, x+\tau_{1}-\tau_{2}, x\right) d x\right\}, t \leq \tau_{2}, \\
\left.\left(\alpha U_{1}\right)\right|_{\left(t-\tau_{2}, \tau_{1}-\tau_{2}\right)} \exp \left\{-\int_{0}^{\tau_{2}} \mu_{3}\left(x+t-\tau_{2}, x+\tau_{1}-\tau_{2}, x\right) d x\right\}, t \geq \tau_{2}
\end{array}\right. \\
U_{4}=\left\{\begin{array}{l}
f_{1}\left(t, \tau_{1}, \tau_{3}\right), t \leq \tau_{3}+T_{0}, \\
\left.\left(\alpha U_{1}\right)\right|_{\left(t-\tau_{3}-T_{0}, \tau_{1}-\tau_{3}-T_{0}\right)} \exp \left\{-\int_{0}^{T_{0}} \mu_{3}\left(x+t-\tau_{3}-T_{0}, x+\tau_{1}-\right.\right. \\
\left.\left.\tau_{3}-T_{0}, x\right) d x-\int_{0}^{\tau_{3}} \mu_{4}\left(x+t-\tau_{3}, x+\tau_{1}-\tau_{3}, x\right) d x\right\}, t \geq \tau_{3}+T_{0}
\end{array}\right.
\end{gathered}
$$

with

$$
\begin{aligned}
& f=\left\{\begin{array}{l}
u_{40}\left(\tau_{1}-t, \tau_{3}-t\right) \exp \left\{-\int_{\tau_{3}-t}^{\tau_{3}} \mu_{4}\left(x+t-\tau_{3}, x+\tau_{1}-\tau_{3}, x\right) d x\right\}, t \leq \tau_{3}, \\
u_{30}\left(\tau_{1}-t, T_{0}+\tau_{3}-t\right) \exp \left\{-\int_{T_{0}+\tau_{3}-t}^{T_{0}} \mu_{3}\left(x+t-\tau_{3}-T_{0}, x+\tau_{1}-\right.\right. \\
\left.\left.\tau_{3}-T_{0}, x\right) d x-\int_{0}^{\tau_{3}} \mu_{4}\left(x+t-\tau_{3}, x+\tau_{1}-\tau_{3}, x\right) d x\right\}, \tau_{3} \leq t \leq \tau_{3}+T_{0} ;
\end{array}\right. \\
& \qquad \begin{array}{l}
u_{10}\left(\tau_{1}-t\right) \exp \left\{-\int_{\tau_{1}-t}^{\tau_{1}} \tilde{\mu}_{1}\left(x+t-\tau_{1}, x\right) d x\right\}+\int_{\tau_{1}-t}^{\tau_{1}} U_{4}\left(y+t-\tau_{1},\right. \\
\left.y, h-T_{0}\right) \exp \left\{-\int_{y}^{\tau_{1}} \tilde{\mu}_{1}\left(x+t-\tau_{1}, x\right) d x\right\} d y, t \leq \tau_{1}-\tau_{1}^{s}, \\
U_{1}\left(\tau_{1}^{s}+t-\tau_{1}, \tau_{1}^{s}\right) \exp \left\{-\int_{\tau_{1}^{s}}^{\tau_{1}} \tilde{\mu}_{1}\left(x+t-\tau_{1}, x\right) d x\right\}+ \\
\int_{\tau_{1}^{s}}^{\tau_{1}} U_{4}\left(y+t-\tau_{1}, y, h-T_{0}\right) \exp \left\{-\int_{y}^{\tau_{1}} \tilde{\mu}_{1}\left(x+t-\tau_{1}, x\right) d x\right\} d y, \\
{\left[U_{1}\left(t, \tau_{1}^{i}\right)\right]=0, t \geq \tau_{1}-\tau_{1}^{s}, \tau_{1} \in\left(\tau_{1}^{s}, \tau_{1}^{s+1}\right], s=0, \ldots, 4, i=1,2,3,4,}
\end{array}
\end{aligned}
$$

where $\tau_{1}^{j}=T_{j}$ for $j=0,1,2, \tau_{1}^{3}=T_{n+1}, \tau_{1}^{4}=T_{n+2}, \tau_{1}^{5}=\infty$, and

$$
\tilde{\mu}_{1}=\left\{\begin{array}{l}
\mu_{1}, \tau_{1} \notin \bar{\sigma}_{1}, \\
\mu_{1}+\alpha, \tau_{1} \in \bar{\sigma}_{1}, \quad U_{4}\left(t, \tau_{1}, h-T_{0}\right)=0 \text { for } \tau_{1} \notin \bar{\sigma}_{2} .
\end{array}\right.
$$

We must add Eq. $(3.2 .6)_{2}$ to system (3.2.10)-(3.2.13). The functions $U_{2}\left(t, \tau_{1}, T_{0}\right)$ and $U_{4}\left(t, \tau_{1}, h-T_{0}\right)$ involved in Eqs. $(3.2 .6)_{2}$ and (3.2.13) are defined as follows:

$$
\begin{aligned}
\left.U_{2}\right|_{\tau_{2}=T_{0}}=\left\{\begin{array}{l}
u_{20}\left(\tau_{1}-t, T_{0}-t\right) \exp \left\{-\int_{T_{0}-t}^{T_{0}} \mu_{23}\left(x+t-T_{0},\right.\right. \\
\left.\left.x+\tau_{1}-T_{0}, x\right) d x\right\}, t \leq T_{0}, \\
\left.\left(\alpha U_{1}\right)\right|_{\left(t-T_{0}, \tau_{1}-T_{0}\right)} b\left(t-T_{0}, \tau_{1}-T_{0}, N\left(t-T_{0}\right)\right) \times \\
\exp \left\{-\int_{0}^{T_{0}} \mu_{23}\left(x+t-T_{0}, x+\tau_{1}-T_{0}, x\right) d x\right\}, t \geq T_{0} ;
\end{array}\right. \\
\left.U_{4}\right|_{\tau_{3}=h-T_{0}}=\left\{\begin{array}{l}
f_{1}\left(t, \tau_{1}, h-T_{0}\right), t \leq h, \\
\left.\left(\alpha U_{1}\right)\right|_{\left(t-h, \tau_{1}-h\right)} \exp \left\{-\int_{0}^{T_{0}} \mu_{3}\left(x+t-h, x+\tau_{1}-h, x\right) d x-\right. \\
\left.\int_{0}^{h-T_{0}} \mu_{4}\left(x+t-h+T_{0}, x+\tau_{1}-h+T_{0}, x\right) d x\right\}, t \geq h
\end{array}\right.
\end{aligned}
$$

Because of the delays $T_{0}$ and $h$ system $(3.2 .6)_{2}$ and (3.2.10)-(3.2.13) has a retarded structure. Equation $(3.2 .13)_{1}$ defines $U_{1}\left(t, \tau_{1}\right)$ for $t \in\left[0, \tau_{1}-T_{0}\right]$ with $\tau_{1} \in\left[T_{0}, T_{1}\right]$. Then from Eq. (3.2.13) we find $U_{1}\left(t, \tau_{1}\right)$ for $t \in\left[0, \tau_{1}-T_{0}\right]$ with $\tau_{1} \in\left[T_{1}, T_{2}\right]$. Due to the delay $h$, by (3.2.15), we determine $U_{4}\left(t, \tau_{1}, h-T_{0}\right)$ for $t \in\left[0, \tau_{1}-T_{0}\right]$ with $\tau_{1} \in\left[T_{2}, T_{3}\right]$. Now going along the axis $\tau_{1}$ by the step $h$, we construct $U_{1}\left(t, \tau_{1}\right)$ for $t \in\left[0, \tau_{1}-T_{0}\right]$ and $\tau_{1} \in\left[T_{0}, T_{n+2}\right]$. Finally, Eq. (3.2.13) defines $U_{1}\left(t, \tau_{1}\right)$ for $t \in\left[0, \tau_{1}-T_{0}\right]$ and $\tau_{1} \in\left[T_{n+2}, \infty\right)$. Then from (3.2.11) and (3.2.12) we find $U_{3}$ and $U_{4}$ for $t \leq \tau_{1}-T_{0}$ and all $\tau_{1}, \tau_{2}$, and $\tau_{3}$. 
It is easy to see that, for $t \geq \tau_{1}-T_{0}$,

$$
\left\{\begin{array}{l}
U_{1}\left(t, \tau_{1}\right)=U_{1}\left(T_{0}+t-\tau_{1}, T_{0}\right) v_{1}\left(t, \tau_{1}\right), v_{1}\left(t, T_{0}\right)=1, \\
U_{3}\left(t, \tau_{1}, \tau_{2}\right)=U_{1}\left(T_{0}+t-\tau_{1}, T_{0}\right) v_{3}\left(t, \tau_{1}, \tau_{2}\right) \\
U_{4}\left(t, \tau_{1}, \tau_{3}\right)=U_{1}\left(T_{0}+t-\tau_{1}, T_{0}\right) v_{4}\left(t, \tau_{1}, \tau_{3}\right)
\end{array}\right.
$$

where $v_{3}, v_{4}$, and $v_{1}$ satisfy Eqs. (3.2.11)-(3.2.13) and (3.2.15) with $U_{i}$ replaced by $v_{i}$. Starting with $v_{1}\left(t, T_{0}\right)=1$ for $t \geq 0$ and using the argument above, we construct $v_{1}, v_{3}$, and $v_{4}$ for $t \in\left[\tau_{1}-T_{0}, \infty\right)$ with all $\tau_{1}$.

It remains to determine $U_{2}\left(t, \tau_{1}, \tau_{2}\right), U_{1}\left(t, T_{0}\right), \beta(t), N(t)$, and $f(t)$ and then, by (3.2.2), find $u_{1}, u_{2}, u_{3}$, and $u_{4}$. Knowing, by $(3.2 .10)_{1}, U_{2}$ for $t \leq \tau_{2}$ and hence $U_{2}\left(t, \tau_{1}, T_{0}\right)$ for $t \in\left[0, T_{0}\right]$, from $(3.2 .6)_{2}$ we find $U_{1}\left(t, T_{0}\right) \in C^{0}\left(\left[0, T_{0}\right]\right) \cap C^{1}\left(\left[0, T_{0}\right)\right)$. Then, by $(3.2 .16)$, we define $U_{1}, U_{3}$, and $U_{4}$ for $0 \leq t \leq \tau_{1}$ and all $\tau_{1}$ and, by $(3.2 .3), \beta(t) \in C^{1}\left(\left[0, T_{0}\right]\right)$. Now, by (3.2.4) and (3.2.5) we define $N \in C^{1}\left(\left[0, T_{0}\right]\right)$ and $f \in C^{1}\left(\left[0, T_{0}\right]\right)$, respectively. Finally, Eq. (3.2.10) defines $U_{2}$ for $0 \leq t \leq T_{0}+\tau_{2}$ and all $\tau_{1}$ and $\tau_{2}$. Since $U_{2}\left(t, \tau_{1}, T_{0}\right)$ is known for $t \in\left[0,2 T_{0}\right]$, from $(3.2 .6)_{2}$ we find $U_{1}\left(t, T_{0}\right) \in C^{0}\left(\left[T_{0}, 2 T_{0}\right]\right) \cap C^{1}\left(\left(T_{0}, 2 T_{0}\right]\right)$. Then from (3.2.16) we find $U_{1}, U_{3}$, and $U_{4}$ for $t \in\left[\tau_{1}, T_{0}+\tau_{1}\right]$ and all $\tau_{1}$. Repeating the procedure above, we construct $U_{1}, U_{2}, U_{3}, U_{4}, f, N$, and, by (3.2.2), $u_{1}, u_{2}, u_{3}$, and $u_{4}$ for $t \in\left[0, t_{*}\right]$. It is easy to see that the functions $u_{1}, u_{2}, u_{3}$, and $u_{4}$ are continuous everywhere and $C^{1}$-functions in the domains given in theorem. This completes the proof.

3.3. The asymptotic behavior of the general solution. In this section we examine problem (3.1)-(3.7) and (3.1.1) with general initial distributions and study the large time behavior of its solution.

TheOREM 3. Let constants $T_{0}<h<T_{1}$ and functions $u_{10}, \ldots, u_{40}, \mu_{1}, \ldots, \mu_{4}, \alpha$, and $b$ be positive and: $\alpha$ and $b \in C^{1}\left(\bar{\sigma}_{1}\right), \mu_{1} \in C^{1}\left(\bar{D}_{1}\right), \mu_{2}, \mu_{3} \in C^{1,0}\left(\bar{D}_{2}\right), \mu_{4} \in C^{1,0}\left(\bar{D}_{3}\right), u_{10} \in$ $C^{1}\left(\bar{D}_{1}\right) \cap L^{1}\left(D_{1}\right)$ and $u_{10}^{\prime} \in L^{1}\left(D_{1}\right), u_{20}, u_{30} \in C^{1}\left(\bar{D}_{2}\right), u_{40} \in C^{1}\left(\bar{D}_{3}\right)$. Then the large time behavior of the solution of the linear problem (3.2.6)-(3.2.9), which in the case under consideration does not involve $N$, is given by the following:

$$
U_{i} \sim \tilde{U}_{i}=\eta v_{i} \exp \left\{\lambda_{0}\left(T_{0}+t-\tau_{1}\right)\right\}, i=1,2,3,4,
$$

where the constant $\eta$ is determined in (3.3.5), $v_{1}\left(\tau_{1}\right), v_{2}\left(\tau_{1}, \tau_{2}\right), v_{3}\left(\tau_{1}, \tau_{2}\right)$, and $v_{4}\left(\tau_{1}, \tau_{3}\right)$ are defined by Eqs. (3.1.8)-(3.1.11), and $\lambda_{0}$ is the unique real root of (3.1.3) satisfying (3.1.4).

TheOREM 4. Let $\rho$ and $\rho^{\prime}$ be positive for all $N>0$ and $\rho \in C^{1}([0, \infty))$. Then under conditions of Theorem 3 the unique solution of problem (3.1)-(3.7) and (3.1.1) has the following large time asymptotics:

$$
u_{i} \sim v_{i} N(t)(\tilde{\beta})^{-1} \exp \left\{-\lambda_{0} \tau_{1}\right\}, i=1,2,3,4,
$$

with

$$
\begin{aligned}
\tilde{\beta}= & \int_{D_{1}} v_{1}\left(\tau_{1}\right) \exp \left\{-\lambda_{0} \tau_{1}\right\} d \tau_{1}+\int_{D_{2}} v_{2}\left(\tau_{1}, \tau_{2}\right) \exp \left\{-\lambda_{0} \tau_{1}\right\} d \tau_{1} d \tau_{2} \\
& +\int_{D_{3}} v_{3}\left(\tau_{1}, \tau_{3}\right) \exp \left\{-\lambda_{0} \tau_{1}\right\} d \tau_{1} d \tau_{3}
\end{aligned}
$$


and $N(t)$ the unique solution of

$$
N^{\prime}=N\left(\lambda_{0}-\rho(N)\right), N(0)=\widetilde{N}_{0}=\widetilde{\beta} \eta \exp \left\{\lambda_{0} T_{0}\right\}
$$

Moreover, for $i=1,2,3,4$,

$$
\begin{aligned}
& u_{i} \underset{t \rightarrow \infty}{\longrightarrow} 0 \text { if } \lambda_{0} \leq \rho(0), \\
& u_{i} \underset{t \rightarrow \infty}{\longrightarrow} \infty \text { if } \sup _{N \geq 0} \rho(N)<\lambda_{0}<\infty, \\
& u_{i} \underset{t \rightarrow \infty}{\longrightarrow} v_{i} N_{*}(\tilde{\beta})^{-1} \exp \left\{-\lambda_{0} \tau_{1}\right\} \text { if } \lambda_{0}>0 \text { and there } N_{*}>0 \text { such that } \rho\left(N_{*}\right)=\lambda_{0} .
\end{aligned}
$$

Proof of Theorem 3. In the case under consideration $U_{1}, U_{2}, U_{3}$, and $U_{4}$ of Sect. 3.2, for $t \geq \tau_{1}-T_{0}$, can be written as follows:

$$
U_{i}=U_{1}\left(T_{0}+t-\tau_{1}, T_{0}\right) v_{i}, i=1,2,3,4,
$$

where $U_{1}\left(t, T_{0}\right)$ is defined by Eq. $(3.2 .6)_{2}$. Now we find an upper bound for $U_{1}\left(t, T_{0}\right)$. It follows from $(3.2 .6)_{2}$ that

$$
U_{1}\left(t, T_{0}\right)=\left\{\begin{array}{l}
\int_{\sigma} U_{2}\left(t, \tau_{1}, T_{0}\right) d \tau_{1}, 0 \leq t \leq T_{1} \\
\int_{T_{1}+T_{0}}^{t+T_{0}} U_{1}\left(T_{0}+t-\tau_{1}, T_{0}\right) v_{2}\left(\tau_{1}, T_{0}\right) d \tau_{1}+ \\
\int_{t+T_{0}}^{T_{n+1}+T_{0}} u_{2}\left(t, \tau_{1}, T_{0}\right) d \tau_{1}, t \in\left[T_{1}, T_{n+1}\right] \\
\int_{\sigma} U_{1}\left(T_{0}+t-\tau_{1}, T_{0}\right) v_{2}\left(\tau_{1}, T_{0}\right) d \tau_{1}, t \geq T_{n+1} .
\end{array}\right.
$$

Hence

$$
U_{1}\left(t, T_{0}\right) \leq \xi \int_{t-T_{n+1}}^{t-T_{1}} U_{1}\left(x, T_{0}\right) d x, \xi=\sup _{\tau_{1} \in \bar{\sigma}_{2}} v_{2}\left(\tau_{1}, T_{0}\right), t \geq T_{n+1}
$$

Now

$$
U_{1}\left(t, T_{0}\right) \leq \eta \xi, \eta=\int_{0}^{T_{n+1}} U_{1}\left(x, T_{0}\right) d x, t \in\left[T_{n+1}, T_{n+1}+T_{1}\right]
$$

then

$$
\begin{aligned}
U_{1}\left(t, T_{0}\right) & \leq \xi \int_{T_{1}}^{T_{n+1}+T_{1}} U_{1}\left(x, T_{0}\right) d x \leq \xi \int_{T_{1}}^{T_{n+1}} U_{1}\left(x, T_{0}\right) d x+\xi \int_{T_{n+1}}^{T_{n+1}+T_{1}} U_{1}\left(x, T_{0}\right) d x \\
& \leq \xi \eta\left(1+T_{1} \xi\right), \int_{T_{1}}^{T_{n+1}+T_{1}} U_{1}\left(x, T_{0}\right) d x \leq \eta\left(1+T_{1} \xi\right), t \in\left(T_{n+1}+T_{1}, T_{n+1}+2 T_{1}\right],
\end{aligned}
$$

and, by induction,

$$
U_{1}\left(t, T_{0}\right) \leq \xi \int_{m T_{1}}^{T_{n+1}+m T_{1}} U_{1}\left(x, T_{0}\right) d x \leq \xi \eta\left(1+T_{1} \xi\right)^{m}, t \in\left(T_{n+1}+m T_{1}, T_{n+1}+(m+1) T_{1}\right] .
$$

Therefore there exists the Laplace transform $f\left(\lambda, T_{0}\right)$ of $U_{1}\left(t, T_{0}\right)$,

$$
\begin{aligned}
f\left(\lambda, T_{0}\right)= & \int_{0}^{\infty} U_{1}\left(t, T_{0}\right) \exp \{-t \lambda\} d t=\int_{\sigma} d \tau_{1} \int_{0}^{\tau_{1}-T_{0}} U_{2}\left(t, \tau_{1}, T_{0}\right) \exp \{-t \lambda\} d t \\
& +\int_{\sigma} d \tau_{1} \int_{\tau_{1}-T_{0}}^{\infty} U_{1}\left(T_{0}+t-\tau_{1}, T_{0}\right) v_{2}\left(\tau_{1}, T_{0}\right) \exp \{-t \lambda\} d t \\
= & I(\lambda)+R(\lambda) f\left(\lambda, T_{0}\right),
\end{aligned}
$$


where

$$
I(\lambda)=\int_{\sigma} d \tau_{1} \int_{0}^{\tau_{1}-T_{0}} U_{2}\left(t, \tau_{1}, T_{0}\right) \exp \{-t \lambda\} d t, R(\lambda)=\int_{\sigma} v_{2}\left(\tau_{1}, T_{0}\right) \exp \left\{\lambda\left(T_{0}-\tau_{1}\right)\right\} d \tau_{1} .
$$

Hence

$$
f\left(\lambda, T_{0}\right)=I(\lambda)(1-R(\lambda))^{-1}
$$

Roots of $R(\lambda)$ are discussed in Sect. 3.1. The function $I(\lambda)$ is analytic and using the method of a rectangle contour integral (see [1]), we evaluate the inverse Laplace transform obtaining

$$
U_{1}\left(t, T_{0}\right) \sim \tilde{U}_{1}\left(t, T_{0}\right):=\eta \exp \left\{t \lambda_{0}\right\}, \eta=-I\left(\lambda_{0}\right) / R^{\prime}\left(\lambda_{0}\right)
$$

with $\lambda_{0}$ the unique real root of Eq. (3.1.3) satisfying (3.1.4). Now, by (3.3.3), (3.3.1) follows.

Proof of Theorem 4. Differentiating (3.2.3) and (3.2.6) $)_{2}$ with respect to $t$ and taking into account the continuity of $U_{1}, U_{2}, U_{3}$, and $U_{4}$ at the sets of discontinuity of $\partial_{t} U_{1}, \ldots, \partial_{t} U_{4}$, we get

$$
\beta^{\prime}=\int_{D_{1}} \partial_{t} U_{1} d \tau_{1}+\int_{D_{2}} \partial_{t} U_{3} d \tau_{1} d \tau_{2}+\int_{D_{3}} \partial_{t} U_{4} d \tau_{1} d \tau_{3}
$$

and

$$
U_{1}^{\prime}\left(t, T_{0}\right)=\int_{\sigma} \partial_{t} U_{2}\left(t, \tau_{1}, T_{0}\right) d \tau_{1}
$$

The method used to derive Eqs. (3.3.4) and (3.3.5) may be applied to Eq. (3.3.7) to obtain

$$
\begin{gathered}
\int_{0}^{\infty} U_{1}^{\prime}\left(t, T_{0}\right) \exp \{-t \lambda\} d t=I_{1}(\lambda)(1-R(\lambda))^{-1} \\
I_{1}(\lambda)=\int_{\sigma} d \tau_{1} \int_{0}^{\tau_{1}-T_{0}} \partial_{t} U_{2}\left(t, \tau_{1}, T_{0}\right) \exp \{-t \lambda\} d t .
\end{gathered}
$$

Hence, for large time,

$$
U_{1}^{\prime}\left(t, T_{0}\right) \sim-I_{1}\left(\lambda_{0}\right)\left(R^{\prime}\left(\lambda_{0}\right)\right)^{-1} \exp \left\{t \lambda_{0}\right\}
$$

where, by (3.3.3) with $i=2,(3.1 .3)$, and $(3.2 .6)_{2}$ with $t=0$, $I_{1}\left(\lambda_{0}\right)=\int_{\sigma}\left\{U_{2}\left(\tau_{1}-T_{0}, \tau_{1}, T_{0}\right) \exp \left\{\lambda_{0}\left(T_{0}-\tau_{1}\right)\right\}-U_{2}\left(0, \tau_{1}, T_{0}\right)\right\} d \tau_{1}+\lambda_{0} I\left(\lambda_{0}\right)=\lambda_{0} I\left(\lambda_{0}\right)$. This and (3.3.5) give

$$
U_{1}^{\prime}\left(t, T_{0}\right) \sim \lambda_{0} U_{1}\left(t, T_{0}\right)
$$

and, by (3.3.6) and (3.3.2), $\beta^{\prime} / \beta \sim \lambda_{0}$ for large time. Therefore Eq. (3.3.2) is the asymptotic form of (3.2.4). Finally, from Eqs. (3.3.1), (3.2.3), (3.2.5), (3.2.2), and (3.3.2) the conclusion follows. 
4. The dispersing population dynamics model. In this section we generalize the model in Sect. 3 by including random spatial diffusion in $\Omega$, an open bounded domain in $\mathbb{R}^{m}$ with extremely inhospitable boundary $\partial \Omega$ (or without migration across it), and examine separable and steady-state solutions in the case of constant diffusion modulus $\kappa$ and stationary vital rates.

The general model reads as follows:

$$
\begin{aligned}
& \left\{\begin{array}{l}
\mathfrak{D}_{1} u_{1}-\kappa \Delta u_{1}=-\nu_{1}\left(t, \tau_{1}, x, N\right) u_{1}- \\
\left\{\begin{array}{l}
0, \tau_{1} \notin \bar{\sigma}_{1}, \\
\alpha\left(t, \tau_{1}, x, N\right) u_{1}, \tau_{1} \in \sigma_{1}
\end{array}+\left\{\begin{array}{l}
0, \tau_{1} \notin \bar{\sigma}_{2}, \\
\left.u_{4}\right|_{\tau_{3}=h-T_{0}}, \tau_{1} \in \sigma_{2},
\end{array} \tau_{1} \in D_{1}, x \in \Omega, t>0,\right.\right. \\
\left.u_{1}\right|_{\tau_{1}=T_{0}}=\left.\int_{\sigma} u_{2}\right|_{\tau_{2}=T_{0}} d \tau_{1}, t \geq 0, x \in \bar{\Omega}, \\
\left.u_{1}\right|_{t=0}=u_{10} \text { in } \bar{D}_{1} \times \bar{\Omega}, \\
{\left[\begin{array}{l}
\left.u_{1}\right|_{\tau_{1}=T_{s}}=0, t \geq 0, x \in \bar{\Omega}, s=1,2, n+1, n+2, \\
\left.\mathcal{B} u_{1}\right|_{\partial \Omega}=0, t \geq 0, \tau_{1} \in \bar{D}_{1} ;
\end{array}\right.}
\end{array}\right. \\
& \left\{\begin{array}{l}
\mathfrak{D}_{2} u_{2}-\kappa \Delta u_{2}=-\left(\nu_{2}+\nu_{3}\left(t, \tau_{1}, \tau_{2}, x, N\right)\right) u_{2},\left(\tau_{1}, \tau_{2}\right) \in D_{2}, x \in \Omega, t>0, \\
\left.u_{2}\right|_{\tau_{2}=0}=\alpha\left(t, \tau_{1}, x, N\right) b\left(t, \tau_{1}, x, N\right) u_{1}, \tau_{1} \in \bar{\sigma}_{1}, t \geq 0, x \in \bar{\Omega}, \\
\left.u_{2}\right|_{t=0}=u_{20} \text { in } \bar{D}_{2} \times \bar{\Omega}, \\
\left.\mathcal{B} u_{2}\right|_{\partial \Omega}=0, t \geq 0,\left(\tau_{1}, \tau_{2}\right) \in \bar{D}_{2} ;
\end{array}\right. \\
& \left\{\begin{array}{l}
\mathfrak{D}_{2} u_{3}-\kappa \Delta u_{3}=-\nu_{3}\left(t, \tau_{1}, \tau_{2}, x, N\right) u_{3},\left(\tau_{1}, \tau_{2}\right) \in D_{2}, x \in \Omega, t>0, \\
\left.u_{3}\right|_{\tau_{3}=0}=\alpha\left(t, \tau_{1}, x, N\right) u_{1}, \tau_{1} \in \bar{\sigma}_{1}, x \in \bar{\Omega}, t \geq 0, \\
\left.u_{3}\right|_{t=0}=u_{30} \text { in } \bar{D}_{2} \times \bar{\Omega} \\
\left.\mathcal{B} u_{3}\right|_{\partial \Omega}=0,\left(\tau_{1}, \tau_{2}\right) \in \bar{D}_{2}, t \geq 0
\end{array}\right. \\
& \left\{\begin{array}{l}
\mathfrak{D}_{3} u_{4}-\kappa \Delta u_{4}=-\nu_{4}\left(t, \tau_{1}, \tau_{3}, x, N\right) u_{4},\left(\tau_{1}, \tau_{3}\right) \in D_{3}, x \in \Omega, t>0, \\
\left.u_{4}\right|_{\tau_{3}=0}=\left.u_{3}\right|_{\tau_{2}=T_{0}}, \tau_{1} \in \bar{\sigma}, t \geq 0, x \in \bar{\Omega}, \\
\left.u_{4}\right|_{t=0}=u_{40} \text { in } \bar{D}_{3} \times \bar{\Omega} \\
\left.\mathcal{B} u_{4}\right|_{\partial \Omega}=0,\left(\tau_{1}, \tau_{3}\right) \in \bar{D}_{3}, t \geq 0 ;
\end{array}\right. \\
& N=\int_{D_{1}} u_{1} d \tau_{1}+\int_{D_{2}} u_{3} d \tau_{1} d \tau_{2}+\int_{D_{3}} u_{4} d \tau_{1} d \tau_{3}, t \geq 0, x \in \bar{\Omega},
\end{aligned}
$$

where $\Delta$ is the Laplace operator in $\mathbb{R}^{m}, \mathcal{B}=\gamma_{1}(t, x) \partial_{\nu}+\gamma_{2}(t, x)$ with nonnegative $\gamma_{1}$ and $\gamma_{2}$ and such that $\gamma_{1}+\gamma_{2}>0$ on $\partial \Omega$, and $\partial_{\nu}$ the outward normal derivative on $\partial \Omega$. We emphasize that $\nu_{2}$ does not depend on $N$. Starting with the initial distributions $u_{10}, \ldots, u_{40}$ satisfying corresponding compatibility conditions, we want to describe the evolution of $u_{1}, u_{2}, u_{3}$, and $u_{4}$ satisfying (4.1)-(4.5).

4.1. Separable solutions. In this section we restrict ourselves to the case of constant $\kappa$ and vital rates given by (3.1.1) and seek solutions of Eqs. (4.1)-(4.5) in the form

$$
u_{i}=f(t, x) \bar{u}_{i 0}, f(0, x)=f_{0}(x), \bar{u}_{i 0}=U v_{i} \exp \left\{\lambda_{0}\left(T_{0}-\tau_{1}\right)\right\}, i=1,2,3,4
$$

with a positive constant $U$ and functions $v_{1}, \ldots, v_{4}$ and the constant $\lambda_{0}$ determined in 
Sect. 3 by (3.1.8)-(3.1.11), (3.1.3), and (3.1.4). Direct substitution in (4.1)-(4.5) gives

$$
N=f \beta, \beta=\int_{D_{1}} \bar{u}_{10} d \tau_{1}+\int_{D_{2}} \bar{u}_{20} d \tau_{1} d \tau_{2}+\int_{D_{3}} \bar{u}_{30} d \tau_{1} d \tau_{3},
$$

and

$$
\left\{\begin{array}{l}
\partial_{t} N=\left(\lambda_{0}-\rho(N)\right) N+\kappa \Delta N, t>0, x \in \Omega, \\
N(0, x)=\beta f_{0}(x), x \in \bar{\Omega}, \\
\left.\mathcal{B} N\right|_{\partial \Omega}=0, t \geq 0 .
\end{array}\right.
$$

The solvability of problem (4.1.3) with the homogeneous Dirichlet boundary condition $\left(\gamma_{1}=0, \gamma_{2}=1\right)$ and the asymptotic behavior of its solution are well known (see, e.g., [2], [5], [9], [10]). Let $\Lambda_{1}>0$ be the first eigenvalue of the Dirichlet problem for $-\Delta$ in $\Omega$. The asymptotics of $N$ enables us to formulate

TheOrem 5. Let $\partial \Omega \in C^{2+\delta}, \delta \in(0,1)$ and $f_{0} \in C^{0}(\bar{\Omega})$ be positive in $\Omega$ and $\left.f_{0}\right|_{\partial \Omega}=0$. Then under the conditions of Theorem 1 problem (4.1)-(4.5) and (3.1.1) with the homogeneous Dirichlet boundary condition has a one-parameter class of separable solutions defined by Eqs. (4.1.1)-(4.1.3) and (3.1.8)-(3.1.11). Any separable solution, positive in $\Omega$, is such that

$\left(u_{1}(t, \cdot), u_{2}(t, \cdot), u_{3}(t, \cdot), u_{4}(t, \cdot)\right) \underset{t \rightarrow \infty}{\longrightarrow} 0$ uniformly on $\left(\bar{D}_{1} \times \Omega, \bar{D}_{2} \times \Omega, \bar{D}_{2} \times \Omega, \bar{D}_{3} \times \Omega\right)$ if $\lambda_{0} \leq \kappa \Lambda_{1}+\gamma$ with $\gamma=\inf _{N \geq 0} \rho(N)$. If $\lambda_{0}>\kappa \Lambda_{1}$ and there exists $N_{*}>0$ such that $\rho\left(N_{*}\right)=\lambda_{0}$, then

$$
\left(u_{1}(t, \cdot), u_{2}(t, \cdot), u_{3}(t, \cdot), u_{4}(t, \cdot)\right) \underset{t \rightarrow \infty}{\longrightarrow}\left(\bar{u}_{10}, \bar{u}_{20}, \bar{u}_{30}, \bar{u}_{40}\right) \tilde{N} / \beta
$$

uniformly on $\left(\bar{D}_{1} \times \Omega, \bar{D}_{2} \times \Omega, \bar{D}_{2} \times \Omega, \bar{D}_{3} \times \Omega\right)$, where $\tilde{N}(x)$ is the solution of

$$
-\kappa \Delta \tilde{N}=\tilde{N}\left(\lambda_{0}-\rho(\tilde{N})\right),\left.\quad \tilde{N}\right|_{\partial \Omega}=0,
$$

positive in $\Omega$.

This theorem is analogous to that of Langlais [5] proved for the Gurtin and MacCamy model [3]. Equation (4.1.4) shows that $\lambda_{0}$ is an eigenvalue of the Dirichlet problem for $-\kappa \Delta+\rho(\tilde{N}) I$ in $\Omega$, where $I$ is the identity operator. The structure of separable solutions of model (4.1)-(4.5) and (3.1.1) with the homogeneous Neumann boundary condition $\left(\gamma_{1}=1, \gamma_{2}=0\right)$ is given by

TheOREM 6. Let $T_{0}<h<T_{1}$ and functions $f_{0}, \mu_{1}, \ldots, \mu_{2}, \alpha$, and $b$ be positive, $\rho \in$ $C^{1}\left(\mathbb{R}^{1}\right)$ and such that $\rho>0$ for $N \neq 0$ and $\rho^{\prime}>0$ for $N>0, f_{0} \in C^{0}(\bar{\Omega}),\left.\partial_{\nu} f_{0}\right|_{\partial \Omega}=0$, $\alpha, b \in C^{1}\left(\bar{\sigma}_{1}\right), \mu_{1} \in C^{0}\left(\bar{D}_{1}\right), \mu_{2}, \mu_{3} \in C^{1,0}\left(\bar{D}_{2}\right), \mu_{4} \in C^{1,0}\left(\bar{D}_{3}\right)$, and $\partial \Omega \in C^{2+\delta}, \delta \in$ $(0,1)$. Then problem (4.1)-(4.5) and (3.1.1) with the homogeneous Neumann boundary condition has a one-parameter class of nonnegative separable solutions of type (4.1.1). Any nonnegative (nontrivial) separable solution is such that:

$\left(u_{1}(t, \cdot), u_{2}(t, \cdot), u_{3}(t, \cdot), u_{4}(t, \cdot)\right) \underset{t \rightarrow \infty}{\longrightarrow} 0$ uniformly on $\left(\bar{D}_{1} \times \Omega, \bar{D}_{2} \times \Omega, \bar{D}_{2} \times \Omega, \bar{D}_{3} \times \Omega\right)$ if $\lambda_{0} \leq 0$. 
If $\lambda_{0}>0$ and there exists $N_{*}>0$ such that $\rho\left(N_{*}\right)=\lambda_{0}$, then

$$
\left(u_{1}(t, \cdot), u_{2}(t, \cdot), u_{3}(t, \cdot), u_{4}(t, \cdot)\right) \underset{t \rightarrow \infty}{\longrightarrow}\left(\bar{u}_{10}, \bar{u}_{20}, \bar{u}_{30}, \bar{u}_{40}\right) N_{*} / \beta
$$

uniformly on $\left(\bar{D}_{1} \times \Omega, \bar{D}_{2} \times \Omega, \bar{D}_{2} \times \Omega, \bar{D}_{3} \times \Omega\right)$.

The proof is based on the asymptotic behavior of problem (4.1.3), which for $\rho(N)=$ $a N$ with $a$ a constant is given in Sect. 5.7 of [9]. The asymptotics of $N$ in the general case of $\rho(N)$ can be obtained by the same method.

REMARK 1. Let $u_{10}=f_{0}(x) \tilde{u}_{10}\left(\tau_{1}\right), u_{i 0}=f_{0}(x) \tilde{u}_{i 0}\left(\tau_{1}, \tau_{2}\right), i=2,3, u_{40}=f_{0}(x) \tilde{u}_{40}\left(\tau_{1}\right.$, $\left.\tau_{3}\right)$, where $f_{0}(x)$ satisfies the condition of Theorem $5, \tilde{u}_{j 0}$ satisfies the conditions of Theorem 1 for $u_{j 0}$, and $\mu_{j}, \alpha, b, \rho$ satisfy conditions of Theorem 1 . Then problem (4.1)-(4.5), (3.1.1) with the homogeneous Dirichlet boundary condition has a separable solution of the form $u_{1}=f(t, x) U_{1}\left(t, \tau_{1}\right), u_{i}=f(t, x) U_{i}\left(t, \tau_{1}, \tau_{2}\right), i=2,3, u_{4}=f(t, x) U_{4}\left(t, \tau_{1}, \tau_{3}\right)$, $f(0, x)=f_{0}(x),\left.U_{j}\right|_{t=0}=\tilde{u}_{j 0}, j=1, \ldots, 4$, whose asymptotic behavior can also be determined.

4.2. Steady-state solutions. In this section we consider the stationary case of problem (4.1)-(4.5), (3.1.1). In this case all functions in (4.1)-(4.5), (3.1.1) are independent of $t$ and, hence, $u_{i}=u_{i 0}$ for all $t \geq 0$ with unknown $u_{i 0}$.

TheOREM 7. Let $\partial \Omega \in C^{2+\delta}$. Then under the conditions of Theorem 1 any nontrivial steady-state solution of problem (4.1)-(4.5), (3.1.1) with the homogeneous Dirichlet condition is separable, i.e. any separable solution has the form $\left(u_{1}, \ldots, u_{4}\right)=\left(\bar{u}_{10}, \ldots\right.$, $\left.\bar{u}_{40}\right) \tilde{N} / \beta$ with $\bar{u}_{10}, \ldots, \bar{u}_{40}, \beta$, and $\tilde{N}(x)$ defined in (4.1.1), (4.1.2), and by (4.1.4), respectively.

Proof. We use the Langlais method [5]. Let $u_{1}, \ldots, u_{4}$, and $N$ represent a nontrivial steady-state solution of (4.1)-(4.5) and let $\eta_{j}$ and $w_{j}(x), j \geq 1, w_{1}>0$ in $\Omega$ be the eigenvalues and eigenfunctions of the Dirichlet problem for $-\kappa \Delta w_{j}+\rho(\tilde{N}(x)) I$ in $\Omega$, that is

$$
-\kappa \Delta w_{j}+\rho(\tilde{N}(x)) w_{j}=\eta_{j} w_{j} \text { in } \Omega,\left.\quad w_{j}\right|_{\partial \Omega}=0 .
$$

Then for $u_{1}, \ldots, u_{4} \in L^{2}(\Omega)$ we have

$$
\left\{\begin{array}{l}
u_{1}=\sum_{j=1}^{\infty} u_{1 j} w_{j}(x) v_{1 j}\left(\tau_{1}\right) \exp \left\{\eta_{j}\left(T_{0}-\tau_{1}\right)\right\}, v_{1 j}\left(T_{0}\right)=1, \\
u_{i}=\sum_{j=1}^{\infty} u_{1 j} w_{j}(x) v_{i j}\left(\tau_{1}, \tau_{2}\right) \exp \left\{\eta_{j}\left(T_{0}-\tau_{1}\right)\right\}, i=2,3, \\
u_{4}=\sum_{j=1}^{\infty} u_{1 j} w_{j}(x) v_{4 j}\left(\tau_{1}, \tau_{3}\right) \exp \left\{\eta_{j}\left(T_{0}-\tau_{1}\right)\right\}
\end{array}\right.
$$

where the constants $u_{1 j}$ and functions $v_{s j}$ are to be determined. Direct substitution of (4.2.2) into (4.1)-(4.5) gives Eqs. (3.1.7) with $v_{s}$ replaced by $v_{s j}$ and equations $u_{1 j}\{1-$ $\left.\int_{\sigma} v_{2 j}\left(\tau_{1}, T_{0}\right) \times \exp \left\{\eta_{j}\left(T_{0}-\tau_{1}\right)\right\} d \tau_{1}\right\}=0$ for $u_{1 j}$. Solving these equations for $v_{s j}$, we get $v_{s j}=v_{s}$ with $v_{s}$ defined by (3.1.8)-(3.1.11). Therefore equations for $u_{1 j}$ become

$$
u_{1 j}\left\{1-\int_{\sigma} v_{2 j}\left(\tau_{1}, T_{0}\right) \exp \left\{\eta_{j}\left(T_{0}-\tau_{1}\right)\right\} d \tau_{1}\right\}=0 .
$$

This means that either $u_{1 j}=0$ or $\eta_{j}=\lambda_{0}$, and we conclude that for at most one $j$ we have $u_{1 j} \neq 0$ because (3.1.3) has a unique real root $\lambda_{0}$. For this $j, \eta_{j}=\lambda_{0}$. Thus 
the series (4.2.2) have at most one non-zero term and $u_{1}, \ldots, u_{4}$ represent a separable solution. The proof is complete.

COROLlaRY. When $\lambda_{0}<\kappa \Lambda_{1}+\gamma$ then there exists no nontrivial steady-state solution of the Dirichlet problem for (4.1)-(4.5), (3.1.1).

5. Discussion. In this paper we presented two asexual density-dependent population dynamics models with age-dependence and child care. One of them includes the random diffusion in a bounded domain with an extremely inhospitable boundary (or without migration across it), while in the other the population is assumed to be non-dispersing. In both cases the population consists of the young (under maternal care), juvenile, and adult classes. The analysis is performed for the case when death moduli of the juvenile and adult classes in both models are decomposed into the sum of two terms. The first presents death rate by the natural causes, while the other describes the environmental influence depending on the total size of juvenile and adult classes. It is assumed that young offspring are protected from density related increases of mortality dependent on $N$ directly and that their death rate consists of their natural one independent of $N$ plus the death rate of their mothers. Adult individuals have the reproductive and post-reproductive age grades. Individuals of reproductive age are divided into fertile singles (having no offspring under maternal care at the given time), mothers (taking care of offspring), and temporarily sterile singles (individuals for whom child care time is over but whose age still is in the sterility interval after delivery). Such splitting of the population is natural for some species of animals, that form pairs only for period of mating. For some species the temporarily sterile singles may be neglected.

A global existence and uniqueness result is established for the non-dispersing population model (3.1)-(3.7) with vital rates (3.2.1). The assumption that $\alpha$ is independent of $N$ is caused by technical reasons. From Eqs. (3.2.11)-(3.2.15) and (3.2.3) we see, that involving of $N$ into the set of arguments of $\alpha$ leads to a nonlinear integro-differential equation for $N$ of type (3.2.4) with $\beta$ depending on $N$.

In the case of vital rates of type (3.1.1) the separable solutions of model (3.1)-(3.7) and the long time asymptotics of the unique solution of this model with vital rates (3.1.1) and the general initial distributions are studied. It is shown, that this asymptotics is a separable solution of the same model. It is known (see, e.g., [6], [7], [12], and references there) that this assertion is true for the asexual Sharpe-Lotka-McKendrickvon Foerster model, as well. Any steady-state solution of the dispersing population model (4.1)-(4.5) (as in the case of the asexual Sharpe-Lotka-McKendrick-von Foerster model) with the homogeneous Dirichlet boundary condition has the separable structure.

The child care is included by adding the death rate of mothers to the natural death rate of their offspring and by letting young offspring be protected from the Verhulst density factor. The last assumption is of a technical type which enabled us to split the models of limited population into the models of non-limited one plus equation for $N$ and, of course, let the existence of separable solutions. 


\section{References}

[1] R. Bellman and K. L. Cooke, Differential-Difference Equations, Acad. Press, New YorkLondon, 1963.

[2] C. Dafermos, Asymptotic behavior of solutions of evolution equations, in: Nonlinear Evolution Equations, M. G. Crandal (ed.), Acad. Press, New York, 1978.

[3] M. E. Gurtin and R. MacCamy, Nonlinear age-dependent population dynamics, Arch. Rat. Mech. Anal. 54 (1974), 281-300.

[4] K. P. Hadeler, Pair formation models with maturation period, J. Math. Biol. 32 (1993) $1-15$.

[5] M. Langlais, Large time behavior in a nonlinear age-dependent population dynamics problem with spatial diffusion, J. Math. Biol. 26 (1988), 319-346.

[6] M. Langlais and F. Milner, Separable solutions of an age-dependent population model with age dominance and their stability, Math. Biosci. 119 (1994), 115-124.

[7] M. Martcheva, Exponential growth in age-structured two-sex populations, Math. Biosci. 157 (1999), 1-22.

[8] S. Moss de Oliveira, Consequences of parental care on population dynamics, Physica A 273 (1999), 140-144.

[9] C. V. Pao, Nonlinear Parabolic and Elliptic Equations, Plenum Press, New York, 1992.

[10] D. H. Sattinger, Monotone methods in nonlinear elliptic and parabolic boundary value problems, Indiana University Math. J. 21 (1972), 979-1000.

[11] V. Skakauskas, Two population dynamics models with child care, Informatica (Lithuanian Academy of Sciences) 11 (2000), 195-218.

[12] G. F. Webb, Theory of Non-linear Age-dependent Population Dynamics, New York, 1985. 